\title{
A Simplified Sounding System for Finding NVIS Channel Availability to Support Government Radio Networks in Indonesia
}

\author{
Titon Dutono(*), Zulmi Zakariyah(*), Tribudi Santoso(*), \\ Denny Setiawan(**)
}

(*)Electronic Engineering Polytechnic Institute of Surabaya-EEPIS

$\left({ }^{* *}\right)$ Ministry of Communication and Information Technology-MCIT

titon@pens.ac.id, zulmi.armi@gmail.com, tribudi@pens.ac.id, denny@postel.go.id

\begin{abstract}
Mostly natural disasters in Java Island such as landslides are within the vicinity of not more than $200 \mathrm{Km}$ from the district capital. Cellular communications require complex systems and rather vulnerable to cope with disasters. NVIS mode is considered as a simple radio link during disaster mitigation initiation process. It needs a valid estimation to figure out the condition of the ionosphere. There are two purposes of this study, the first of which is an attempt to find out a fact the existences of authorized HF users who still work in the band of $3 \mathrm{MHz}-10 \mathrm{MHz}$. The second is to integrate low cost $\mathrm{HF}$ radio communication, commonly available small single board computer hardware, and opensource software, to build a sounding system to evaluate the quality of NVIS channels. Prediction system such VOACAP give hourly prediction data, however it has an inherent limitation because of nature the underlying databases is monthly average based, therefore, the estimation could not be made in a daily basis. However, a real-time channel evaluation (RTCE) able to purify maximum observed frequency (MOF) estimation, and consequently, its able to select the best available frequency for short term and real time operation. In this study, we used WSPR to perform a simple RTCE technique. Furthermore, we also reviewed the current regulatory status regarding the availability of sub-10 $\mathrm{MHz}$ band for NVIS radio operation. The results show that discrepancies between simulation and measurement are occurred mainly because of sporadic data in the band of $60 \mathrm{~m}$ and $80 \mathrm{~m}$. However, all of the measurement results and simulations almost have the same agreement regarding the quiet period between local midnight and local sunrise. The results of measurements show that $60 \mathrm{~m}$ band is the most reliable NVIS channel between local sunrise and local midnight. Furthermore, 100 watts is a proper transmitter power to reach the required SNR for reliable voice communication.
\end{abstract}

Keywords: WSPR, beacon, ITU-R, ionosphere, RTCE 


\section{INTRODUCTION}

Cellular telecommunication is considered to have been able to provide telecommunications services in all places on earth. But in fact, there are still many rural areas with geographical terrain covered in mountains, which still cannot be served because the radio waves are obstructed by geographical contours. These areas are commonly called blank spots. Java Island is the main island in the archipelago of Indonesia and it is an island with a densely populated. This island has a good soil because it consists of a cluster of mountains. Nevertheless, this island is almost considered to be overexploitation for the purposes of agricultural land and plantation. So that when the rainy season arrives, landslide can be regarded as an annual disaster that always decorate the news. More than that Java Island is included in the cluster of rings of fire; therefore, the earthquake disaster becomes a thing to watch out for at any time by preparing disaster mitigation in the communication management sector and logistics distribution. Often, we hear that disaster in a rural area is a bit slow implementation of its mitigation because of the problem of disconnection of radio communication network.

Communication support between the Ground-Zero (covered countryside) with the nearest town on D-day plus the first week, just before the recovering of cellular networks, is crucial to determining effective mitigation patterns in order to carry out initial recovery implementation properly.

Cellular communications systems do offer ease, but these systems require complex and fragile support systems to cope with disasters. Furthermore, satellite phone networks are indeed able to overcome blank spot, but the satellite phone system is still so expensive that it cannot be owned massively by all disaster mitigation task force.

The HF or High Frequency band is spread between $3 \mathrm{MHz}$ up and $30 \mathrm{MHz}$ Radio waves with frequency of VHF (Very High Frequency), UHF (Ultra High Frequency) or higher will pass along the atmosphere toward space, without change of direction, other than attenuation due to propagation only. Radio waves on the HF band passes through the ionosphere layers, and under certain conditions the HF radio waves will gradually change the direction until finally back to the earth. Therefore, ionosphere layer is a kind of reflective glass to the HF radio wave. Furthermore, there are four modes of HF propagation, namely: ground wave, direct wave, long range sky wave, and NVIS (Near Vertical Incident Sky wave) [1][2].

Mostly natural disasters in Java Island such as landslides are within the vicinity of not more than $200 \mathrm{Km}$ from the district capital. For this purpose, NVIS mode is considered as an important radio link during disaster mitigation initiation process.

Public protection radiocommunication is radiocommunication used by authorized agencies and organizations in the framework of maintenance of law and order, life protection and property and emergency situations. This statement is stipulated in Resolution 646 of World Radio Conference 2012 (WRC-12) [3]. Further, it is known that current public protection and disaster 
relief applications are narrow-band for supporting voice and low data-rate applications, typically in channel bandwidths of $25 \mathrm{kHz}$ or less [3].

Some people might argue that HF radio is being an obsolete technology, even though HF radio remains a vital communication tool. The progress of the micro-electronics makes modern HF radio systems include voice and digital modulation schemes for the reliable and secure transfer of encrypted data could be realized. Especially government and public interest continue to use advanced HF radio techniques in a variety of novel ways that directly impact on the lives of ordinary people.

NVIS channels availability in HF band needs the occurring of ionization in the ionosphere above the transmitter. It is predicted the numbers of sunspots (SSN) to be the lowest in its cycle period during the current two-year period. SSN is considered has strongly correlated with ionization in the Earth's atmosphere. Therefore, the correlation between SSN and the propagation quality for both NVIS and long-range modes is an interesting matter to be studied during this period.

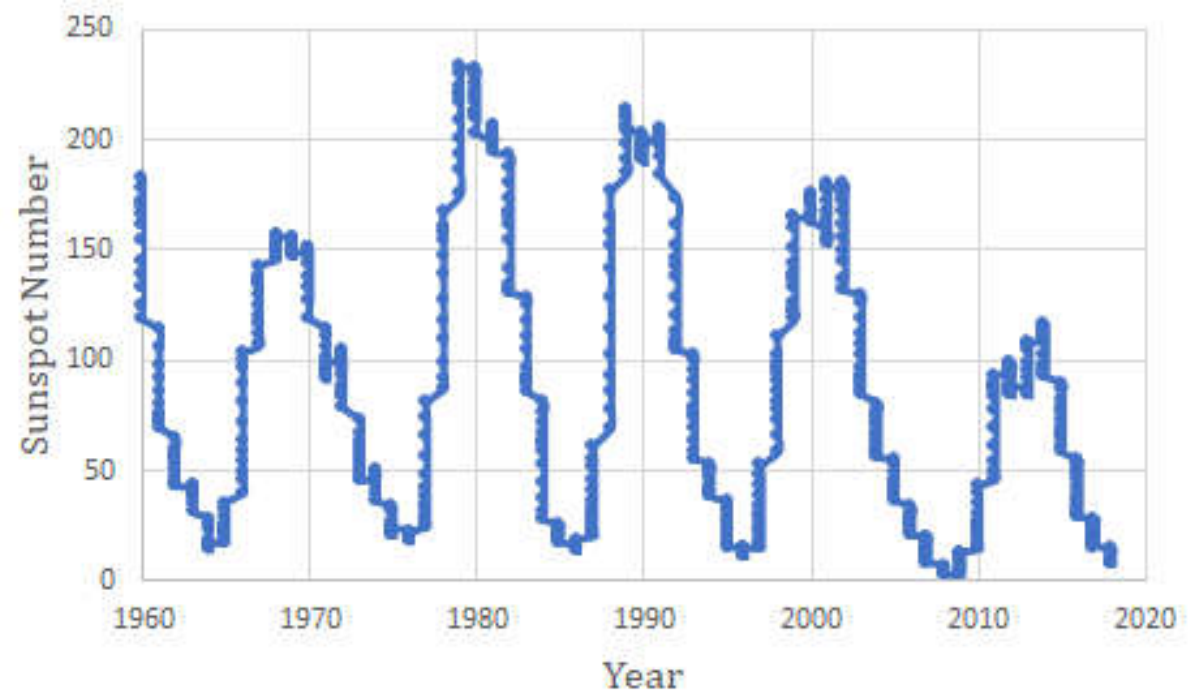

Figure 1. International sunspot number from 1960 until the current condition. It is represented by means of 13-month smoothed number of monthly-mean values.

Figure. 1 represent sunspot number by means of 13-month smoothed number of monthly-mean values, in which the data is downloaded from SILSO (http://sidc.be/silso) Royal Observatory of Belgium. It is shown that year of 2018-2019 would be a minimum of sunspot number during last 5 years.

There are two purposes of this study, the first of which is an attempt to find out a fact the existences of authorized HF users who still work in the band of $3 \mathrm{MHz}-10 \mathrm{MHz}$ The second is to integrate low cost HF radio communication, commonly available small single board computer hardware, and opensource software, to build a sounding system to evaluate the quality of NVIS channels. 
The ultimate goal of this study is a recommendation to be addressed to the Administration of Frequency Authority holders in Indonesia to revise or update the frequency assignment and frequency of special allotment for the purposes of Public Protection and Disaster Relief in the band of $3 \mathrm{MHz}-10$ $\mathrm{MHz}$

\section{REGULATORY CONSIDERATIONS}

In the recent years, some continental countries with large areas such as Australia, the United States and Russia have specifically maintained the HF band as an important communication tool for needs including civil aviation services, national defense, police, fire and maritime safety. Although it is known that those countries already have established modern communication network systems.

The prominent usages of the frequency allocation between $3-10 \mathrm{MHz}$ band are amateur service, fixed service, aeronautical mobile service, and maritime mobile service. Amateur service only occupies about $10 \%$ of bandwidth allocation as a primary basis [4]. The remains $90 \%$ of bandwidth allocation are usable based on an authorization or assignment given by administration/government for a radio station to use a radio frequency or radio frequency channel under specified conditions.

The terms of aeronautical mobile service, maritime mobile service is defined in Radio Regulation [6]. The usage of the frequency in those services generally for the purposes of positioning, beacon, voice communication, during normal and distress conditions [6]. Sub-band allocation of transponder frequencies within aeronautical mobile and maritime mobile spectrum allocation will be carried out under the convention of ICAO (International Civil Aviation Organization) and IMO (International Maritime Organization), respectively. Those two International Organization have authority to manage and develop policies to determine specifics frequencies within the band for operational procedure in the domain of aeronautical and maritime, respectively.

Furthermore, Fixed service is a radio communication service between specified fixed points that operated by authorized entities which are given assignment by administration/government.

The use of radio frequency spectrum and satellite orbit shall be based on acquiring a license from the Government. Furthermore, the use of radio frequency spectrum and satellite orbit must be in accordance with its allocation and not interfere with each other [4][5]. Operators of fixed points radio stations are given an assignment or frequency usage licenses based on the proposal and consideration toward an underlying comprehensive frequency planning.

The Radio Regulations (RR) in which always referred by the Government during frequency planning, contains the complete texts that is adopted by the World Radiocommunication Conference (WRC) and subsequently revised and adopted every 4-years period by World Radiocommunication Conferences (WRC). The RR include all Appendices, 
Resolutions, Recommendations and ITU-R Recommendations incorporated by reference. Frequency spectrum policy and planning are carried out by the Government with the reference of RR. Another consideration is a local wisdom and local need. Even though the same reference is considered, the usage policy to a piece of spectrum frequency may be difference among countries.

In accordance with Resolution 649 of WRC-2012 stated to consider possible new allocation to the amateur service on a secondary basis, to a piece of frequency in the sub-band within the band of $60 \mathrm{~m}$ [3]. Even though in many countries, their governments have already used this band for public protection and disaster relief purposes, this resolution just proposed that this sub-band allow amateur radio service to use this sub-band to a same purpose.

During WRC-2015, concerning this resolution, some countries like Australia and Russia have view to be no change, that means PPDR remain to be a government responsibility. Some countries like USA and China have view that amateur radio could have right to use the band in secondary basis and certain condition. Actually, there are no deferent between those two opposite views, because both views have the same spirit and intend to have the same humanitarian purposes, i.e. PPDR purposes. This point of view shows us that sub-10 MHz HF band have very important and critical usage for PPDR purposes.

WRC always avoid voting among state members during making a decision, instead, WRC always use consensus terminology. The result of Resolution 649 of WRC-2012 is an RR revision of article 5 as footnote 5.113B. This revision is stipulated that stations radio in the amateur service using the frequency band $5351.5-5366.5 \mathrm{kHz}$ shall not exceed a maximum radiated power of 15 watts [6]. In this study, the usage reliability of this band with e.i.r.p 15 watts will be examined by using WSPR beacon system.

\section{FREQUENCY USERS CONSIDERATION}

Frequencies in the range of $3 \mathrm{MHz}-10 \mathrm{MHz}$ empirically usable for near vertical incidence sky wave (NVIS) propagation that allows HF communication over relatively short distances, typically up to $500 \mathrm{~km}$. This communication link is relevance to humanitarian organizations during emergency situations where the normal power and communications infrastructure may have collapsed [1][7]. 


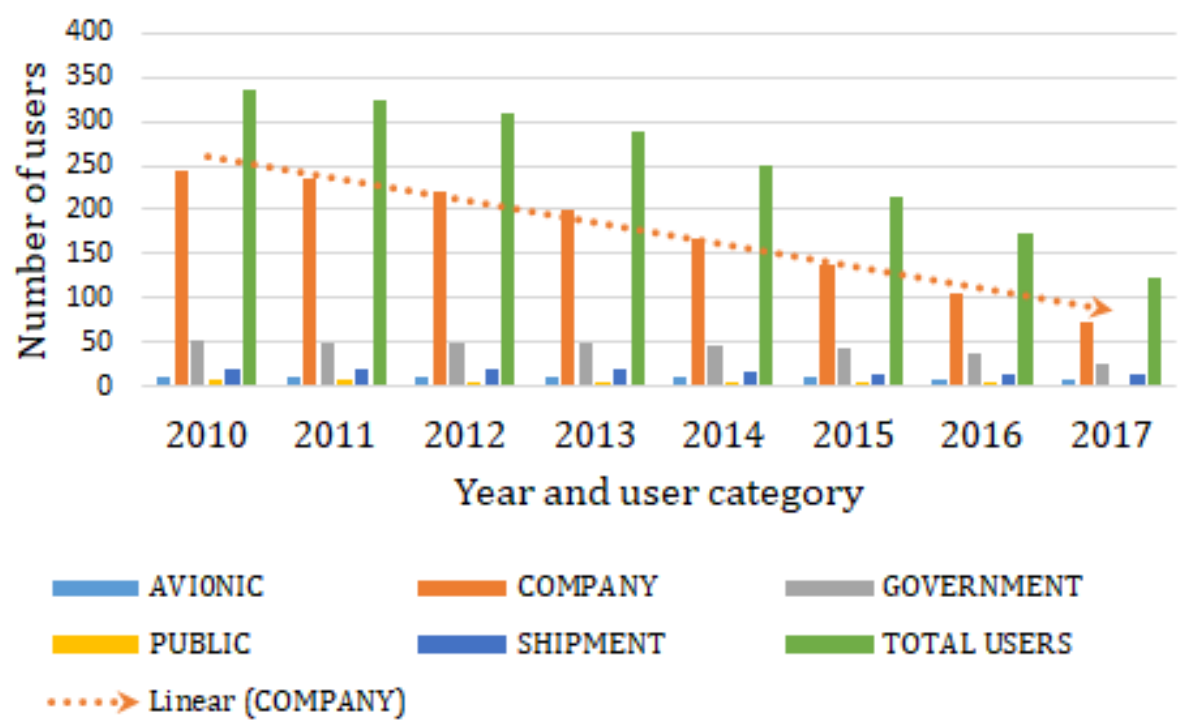

Figure 2. Trend the numbers and category of frequency-band 3-10 MHz users during year $2010-2017^{1}$.

Figure 2 shows the trend in the number of radio frequency usage license holders in the $3-10 \mathrm{MHz}$ band that are still listed on the Database Frequency Register managed by the Indonesian frequency authority (DitjenSDPPI) ${ }^{2}$ since 2010. Those frequencies are used for voice communication with the class emission of a single side band suppressed carrier (J2E). The users occupy those frequencies as legacy, starting when telephony communication was still very expensive. At present in the era of affordable communication, those frequencies are generally no longer used, although some of the users still take care of the licenses every year. The numbers of avionic and shipment users seem to have no change because of the legacy of specific frequency usage in the communication services in the domain of aeronautical and maritime. However, the most prominent users are companies that intend to use these frequencies for fixed long-haul communication radio between their head quarter in Java Island and their branch offices located in eastern part of Indonesia. The Global trend shows that communication prices for terrestrial mobile communications, including mobile satellite communications, are getting affordable. This trend is in line with the trend of declining the number of licenses in this HF domain as shown in Fig.1. The number of company license holders in the year of 2010 is 243 companies, but declining to 73 companies in the year of 2017. It is shown that channel availability in this band is getting better and better. Based on this fact, the Government could decide to make a moratorium in order to stop proceeds from proposals of the usage of this band, instead, this band will be used only for humanitarian usages.

\footnotetext{
${ }^{1}$ By courtesy of Indonesian frequency authority (SDPPI)

${ }^{2}$ Direktorat Jenderal Sumber Daya dan Perangkat Pos dan Informatika
} 


\section{HF IONOSPHERIC PERFORMANCE PREDICTION}

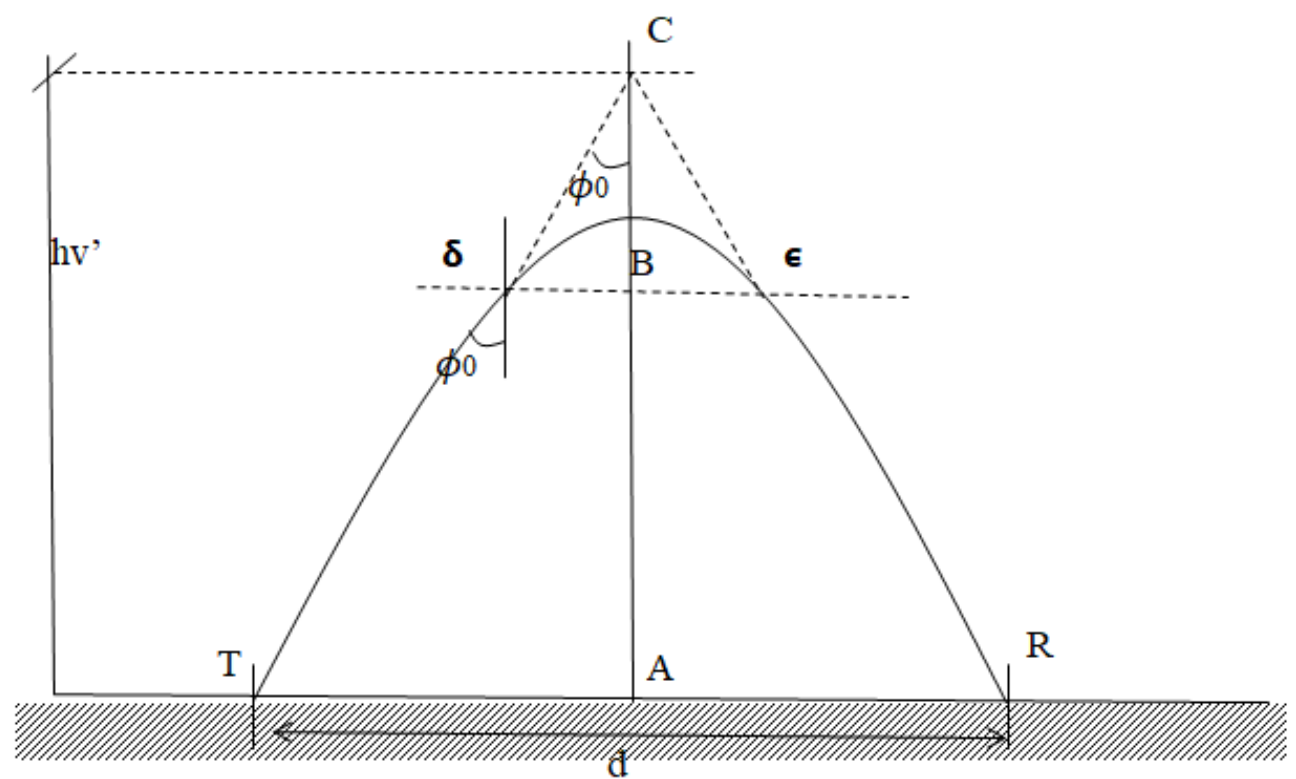

Figure 3. A simplified flat ionosphere model over a simplified flat earth model

The performance of HF radio link estimation depends upon the geophysical condition of ionosphere layers. There are two types of estimation, i.e. (1) geophysical theory based that involve very complicated timeconsuming intensive-computing, (2) measuring based. It is shown in Fig. 3 that any oblique radio frequency $f_{i s}$ will be reflected at the vertical height $h v^{\prime}=C A$, which is called virtual height of a mirror that vertical-reflect a radio signal with frequency $i_{i}[8]$. Therefore

$$
f_{o i}=f_{\mathrm{s}} \sec \left(\phi_{\mathrm{u}}\right)
$$

in which well known as secant law, in which

$$
\sec \left(\phi_{\mathrm{l}}\right)=\sqrt{1+\left(\frac{i \mathrm{i}}{2 n y^{\prime}}\right)^{2}},
$$

where $\mathrm{d}$ is the short path distance between transmitter position $\mathrm{T}$ and receiver position $R$ for the simplified propagation model [8]. NVIS propagation is estimated to propagate via a single hop through the F2 region (IF2), therefore, $h v^{*}$ is virtual height of F2 region. The next problem is the determination value of $d$ based on the given value of $h v^{\prime}$. Over the years, more than 130 sounding/beacon radio stations located widespread all over the world have been collecting the data of ionospheric layers altitude. Statistical and interpolation method is used for arbitrary spot $h v^{\prime}$ estimation. 
VOACAP [9] and REC533 [10] are generally used for most HF propagation study. Both of software carry out prediction that refers to CCIR coefficients. CCIR is former name of ITU-R.

Prediction methods give hourly prediction data. However, they have an inherent limitation because of the nature of underlying databases are average monthly based. Therefore, the estimation could not work in a period of daily bases [11]. However, real-time channel evaluation (RTCE) able to select maximum observed frequency (MOF) estimate, and consequently, its be able to select the best available frequency for short term and real time operation [7].

\section{WSPR BASED SNR MEASUREMENT}

A smart $\mathrm{HF}$ radio is an $\mathrm{HF}$ radio communication unit that able to analyze the propagation condition based on whether instantaneous measurement, or by a prediction. Such kind of radio could be built by attaching the radio with a peripheral device (i.e. small single-board computers-Raspberry Pi) that has a task for signal analyzer sent by the radio.

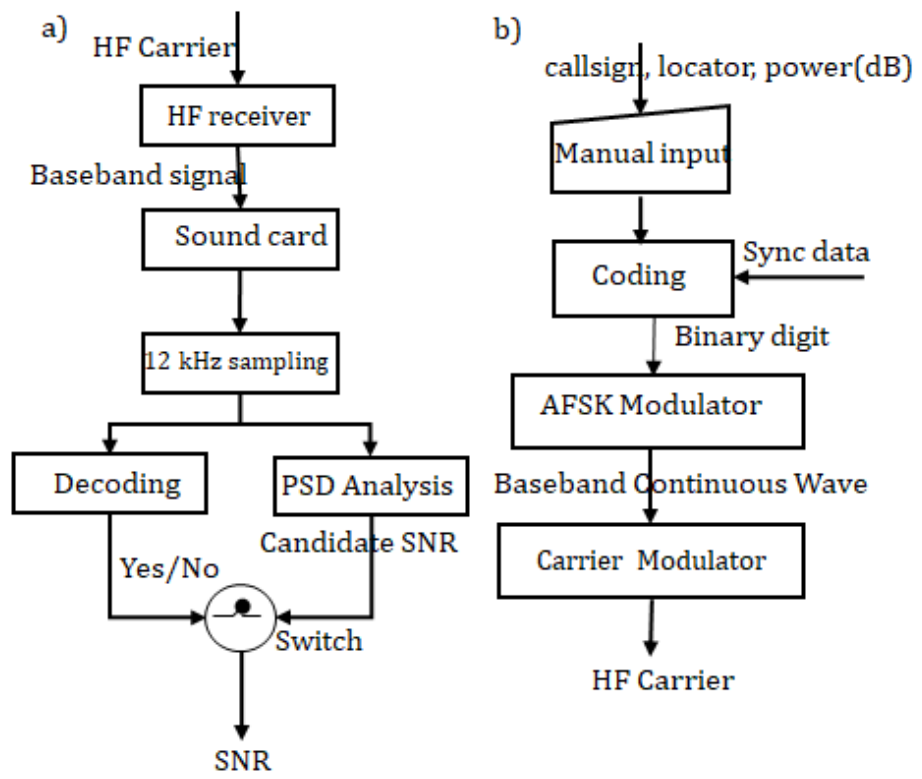

Figure 4. Signal processing in WSPR system

In this section we are going to review at a glance the inside algorithm that make WSPR so reliable [12][13]. The block diagrams of the signal analyzing on the WSPR process are shown in Fig. $4 \mathrm{a}$ and $4 \mathrm{~b}$. The transmitter is shown in Fig. 4b. Set of data consist of call sign, location and transmitted power in $\mathrm{dB}$ are put in to the transmitter. This set of data is then added with redundant bits by using convolutional code. The number of data become 162 bits. The next step is interleaving process. The 162 bits of data are then added with 162 bits of pseudorandom synchronization word. The result is 162 sequential symbols each with 2 bits. Frequency shift of $1.46 \mathrm{~Hz}$ represent every symbol-value giving 4 level Multi-FSK modulation which is 
set to be centered at $1500 \mathrm{~Hz}$. The transmitted period per symbol is approximately 0.684 second. This period is the reciprocal of tone spacing. It means 162 symbols will take about 110,6 seconds with occupied bandwidth of approximately $6 \mathrm{~Hz}$. Finally, the modulation process is carried out with input from AFSK (Audio Frequency Shift Keying) symbol tones.

The receiver is shown in Fig. 4a, firstly, HF carrier is received by the HF radio receiver, then it is demodulated to be a baseband signal. Then the sound card is used for sampling the baseband signals by $12 \mathrm{kHz}$ sampling frequency. This sampling rate is commonly used in the field of speech processing [13]. The baseband continuous wave is an AFSK tones that consist of 162 symbols information of sender station.

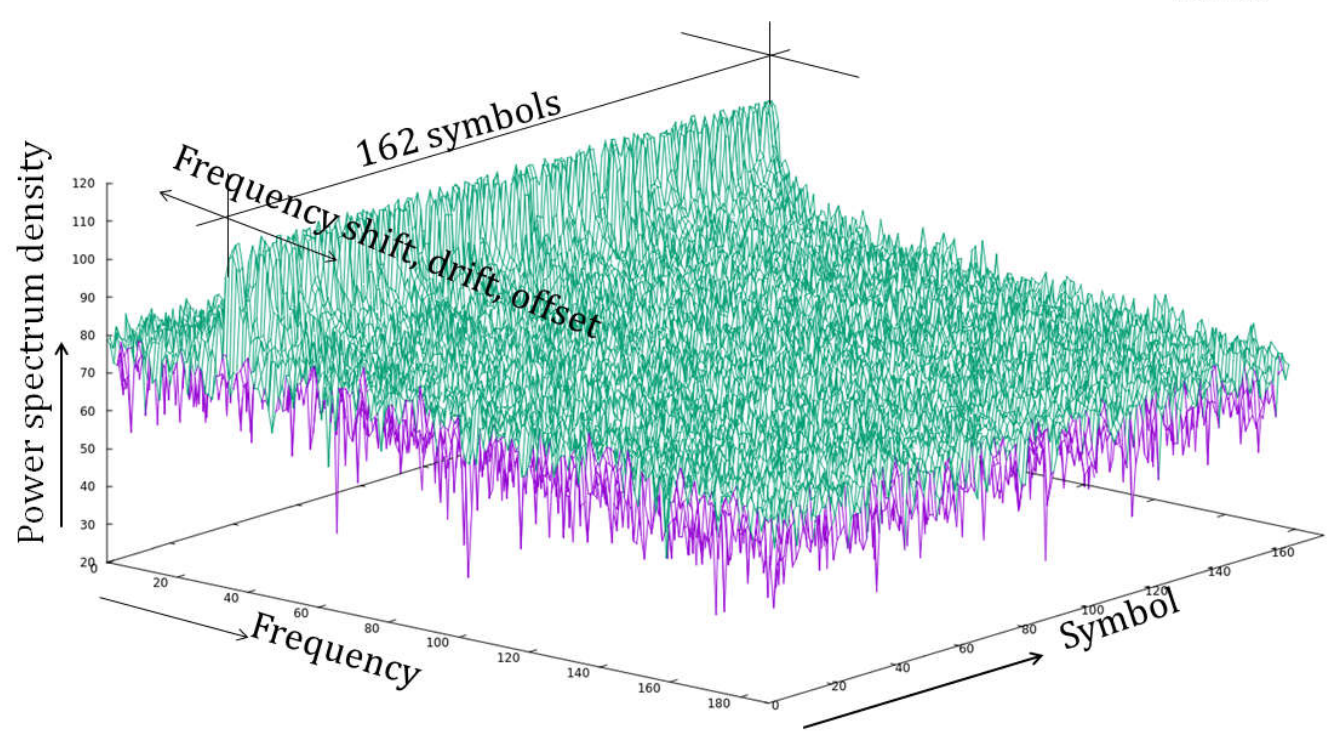

Figure 5. Power spectrum density of tone that consist of 162 symbols; Drift and offset frequency should be removed by using the searching algorithm.

Figure 5 shows a typical 3-d graphic of a single packet of WSPR data. It is shown that frequency drift and offset should be removed from frequency shift that contain of symbol information. When a 2-minute receive interval is accomplished, WSPR starts a process to decode the received signals. Firstly, mixing the received tone with $1500 \mathrm{~Hz}$ local tone, in order to get a new tone with a bandwidth from $1400 \mathrm{~Hz}$ to $1600 \mathrm{~Hz}$, then down- sampling by 1/32 in order to get an extracted tone with the bandwidth of $375 \mathrm{~Hz}$.

Further, the extracted tone is then analyzed its power spectrum in order to find out several candidates of SNR, and to find out several candidates of the center frequencies of tone. Each candidate of center tone frequency corresponds with its SNR candidate.

Let,

$$
A_{i}=\sum_{i} P_{i}\{i\}
$$

where, 
$A$ is average power spectrum density (PSD) across 110.6 second period of tone,

$P$ is PSD of each frame of processing, $j$ is frequency index, across 0 to $375 \mathrm{~Hz}$,

$\dot{i}$ is frame processing index across 110.6 second period of tone.

Furthermore, several candidates are chosen from a local maximum of $\lambda_{i}$ shown in Eq. 4 as follow,

where,

$$
f(n)=\max _{(* n<;<+p: \mid n+1)} A_{j}
$$

$f:(n)$ is n'th candidate center tone frequency across 0 to $375 \mathrm{~Hz}$,

$A$ is average power spectrum density (PSD) across 110.6 second period of tone,

$j$ is frequency index, across 0 to $375 \mathrm{~Hz}$,

$w$ represent the frequency window of approximately $6 \mathrm{~Hz}$.

Each of center tone frequency candidate is then examined by decoding process while removing frequency offset and drift (by maximum $2 \mathrm{~Hz}$ ). The decoding process consists of soft symbols computation, deinterleaving, and decodes the convolutional code using the Fano sequential algorithm. The error rate of decoding process is a clue for SNR candidate to be real or fake.

\section{MEASUREMENT SETUP}

Maximum usable frequency (MUF) and signal strength are approximately independent of the range for communication ranges less than $400 \mathrm{~km} \mathrm{[1][15].} \mathrm{The} \mathrm{points} \mathrm{of} \mathrm{measurement} \mathrm{ware} \mathrm{situated} \mathrm{at} \mathrm{two} \mathrm{cities,} \mathrm{i.e.}$ City of Jombang at the coordinates 7.61S, 112.31E as the point of transmission. Furthermore, the point of receiving was located in the City Surabaya at the coordinates $7.30 \mathrm{~S}, 112.78 \mathrm{E}$ and it located $63 \mathrm{Km}$ away from transmission point. Figure 6 shows the map of measurement points. The radio transmitter power is set to 10 watts, and the antenna is multiple-dipole [16]. The antenna is pulled down its both edges to be an Inverted-V type of antenna, in order to rise up elevation angle of transmission. Furthermore, The receiver side also uses the same type of antenna. NVIS channels use frequencies generally in the range $2-10 \mathrm{MHz}$ [7]. Within the range is then divided into 3 bands of $80 \mathrm{~m}$ band ( $3.5 \mathrm{MHz}), 60 \mathrm{~m}$ band ( $5 \mathrm{MHz}$ ) and $40 \mathrm{~m}$ band $(7 \mathrm{MHz})$. Each band is considered to have their own propagation characteristic. Those bands are amateur services frequency allocations, so every amateur radio license holder may be used the frequency within the bands as far as on a proper operating procedure. The working frequencies are shown in Table.1 
Table 1. Working frequency

\begin{tabular}{|c|c|}
\hline Band & Working frequency \\
\hline $80 \mathrm{~m}$ & $3.5926 \mathrm{MHz}$ \\
\hline $60 \mathrm{~m}$ & $5.2872 \mathrm{MHz}$ \\
\hline $40 \mathrm{~m}$ & $7.0386 \mathrm{MHz}$ \\
\hline
\end{tabular}

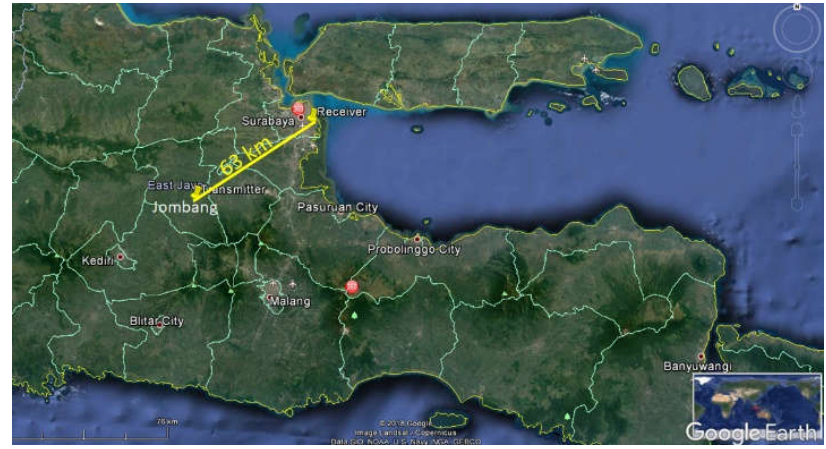

Figure 6. The location of Surabaya as the receiving point and City of Jombang as the transmission point by the distance of $63 \mathrm{Km}$ away.

The operation of WSPR follows the WSPR manual procedure. The radio in transmitter side only carried out transmission. In vice versa the radio in the receiver side only carried out receiving. The measurements have been carried out on three spots of days, i.e. June 17th 2017, October 15th 2017 and January 6th 2018, each for 24 hours. The transmitter is sent the beacon of three working frequency in sequences. It means, the transmitter switched the working frequency every accomplishing sending one packet of WSPR message. The receiver side is set to synchronize the changing of working frequency at the transmitter side. WSPR system software at Surabaya recorded the received SNR. The results of 24 hours measurements, then to be hourly segmented into 24 segment periods. Therefore, each working frequency has several measurement results within one-hour period.

\section{PREDICTION AND MEASUREMENT RESULT COMPARATION}

There are a lot of freeware and proprietary applications that can be used properly for predicting the ionospheric channel. In this study we used freeware VOACAP (http://www.voacap.com) [9].

The only condition affecting the NVIS propagation is the density of electrons of the ionosphere layers that only affected by space weather, instead the surface weather do not affect NVIS wave propagation. VOACAP graphs represent a maximum usable frequency (MUF) in a statistical concept. This graph illustrates the median value of the maximum usable frequency (MUF) attributed with the information of ionospheric paths, hour, day, and Sunspot Numbers (SSN).

NVIS wave obeys the ionospheric path that is reflected by F2 and E layer of ionosphere, in which the existence of F2 layer is predictable, instead 
the E layer also called as sporadic E, just like clouds that their presence and occurrence very difficult to predict [8].

The ionospheric path is predicted based on the prediction of the virtual height $h v^{\prime}$ of ionosphere layer (see Eq. (1), (2)), that is carried out based on retrospective-continues $h v^{\prime}$ measurement (for many years) by more than 130 beacon stations spread all over the world. When a working spot is invoked by a user for $h v^{\prime}$ prediction, then it will be mapped by VOACAP from a location of the nearest beacon station by using a numerical map to obtain, hourly, daily and monthly data of the maximum observation frequency (MOF) [17]. Median value of MOF distribution is represented by the VOACAP graph. Therefore, it can be said that the graph does not represent the maximum usable frequency (MUF) in term of working frequency in radio communication terminology. While the graph of the measurement results is the value of the immediate real observation, which is very dependent on the dynamics of the momentary conditions, such as a local harmful-interference, unpredictable sporadic appearance of ionospheric clouds E layer, and also Solar activity.
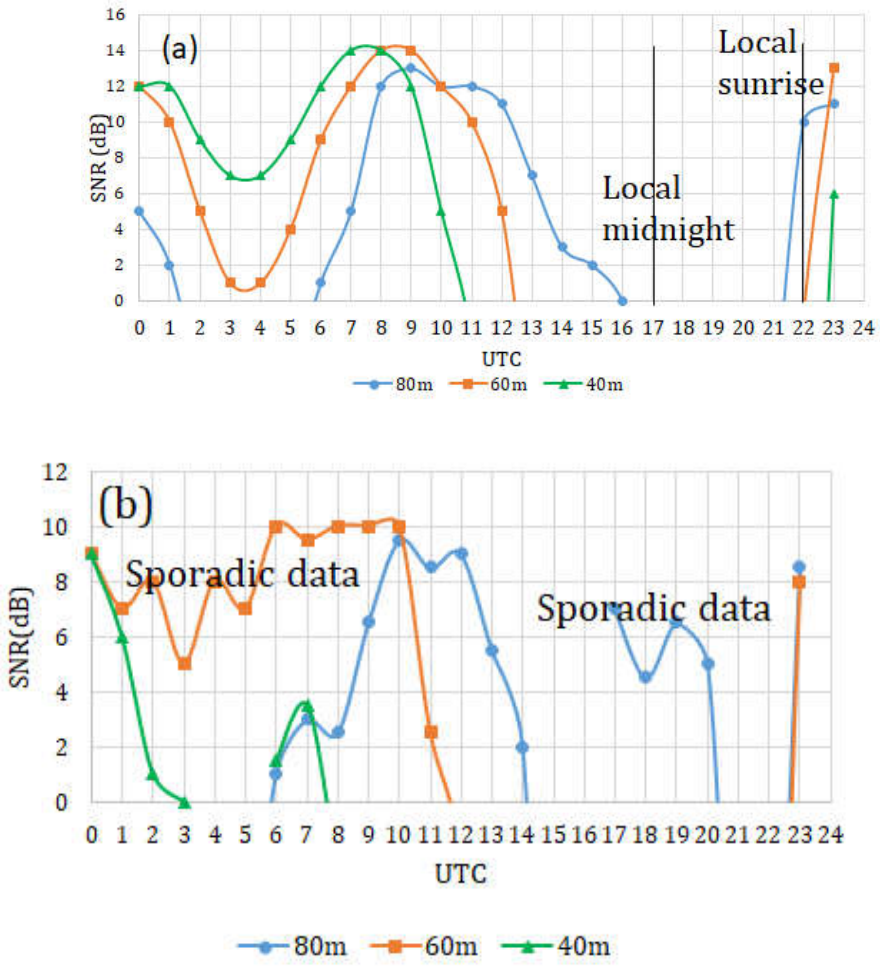

Figure 7. Results of measurement and simulation in June 17th 2017; (a) VOACAP simulation with SSN=19; (b) Measurement results. 

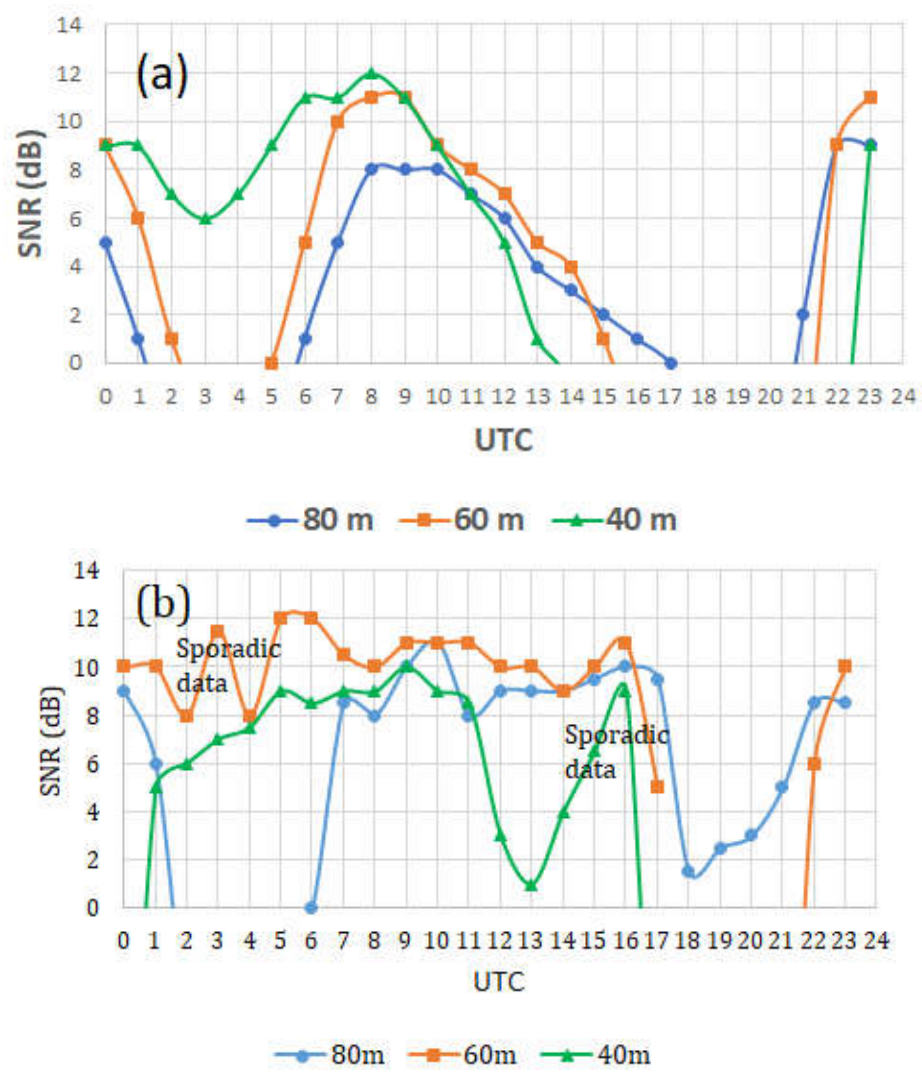

Figure 8. Results of measurement and simulation in October 15th 2017; (a) VOACAP simulation with SSN=13; (b) Measurement results.

The comparative results between the prediction by VOACAP and the observation values are shown in Fig.7(a)(b), Fig.8(a)(b) and Fig.9(a)(b), that depict SNR in dB versus 24 hours UTC. The Figures (a) show the graph of prediction, while, the Figures (b) show the graph of average observations values over 24 hours. Local midnight at 17.00 UTC and local sunrise approximately at 22.00 UTC, are illustrated in Fig. 7(a).

Figure 7(b) depicts the results of measurements on June 17th 2017. It is shown that sporadic data of $60 \mathrm{~m}$ band is detected during morning period, and $80 \mathrm{~m}$ band during midnight. Sporadic data were detected because the appearance of Sporadic E clouds. Furthermore, another sporadic data were also detected during measurements on October 15th 2017. It is shown in Fig. 8 (b) that the sporadic data of $60 \mathrm{~m}$ band are measured during morning period, and $40 \mathrm{~m}$ band during evening period. The same feature of Figure 7,8 and 9 is that the quiet period occurred after local midnight to local sunrise, except a midnight sporadic data of $80 \mathrm{~m}$ band on June 17th 2017 measurement.

Harmful interference causes a failure to measure a received beacon, even though the channel conditions are in good. Let's see the $40 \mathrm{~m}$ band of the graph (green colored) in Figure 7 (b). It is shown that the channel of $40 \mathrm{~m}$ band should be available during UTC 3.00 - 11.00, however, the channel was almost radio blackout during the period. It does not because of ionospheric unavailable, rather because of WSPR failed to decode the beacon due to 
harmful interference by radio amateurs with bad behavior, who do not obey the operating procedures as well as sub-band frequency allocation when using radio frequency. Moreover, the instantaneous local conditions do not always make suffering. Figure 7(b) shows that $80 \mathrm{~m}$ band NVIS channel should be radio blackout during local midnight, but with the rarely, unpredictable appearance of ionosphere E layer clouds, the NVIS channel will sporadic open [8].

Therefore, the VOACAP graph and the measurement results graph cannot be measured in metrics, however by manually subjective, the trends from the two graph representations still be comparable.

VOACAP generally provides accurate estimates of the ionospheric channel [11]. VOACAP simulation results are depicted in figure 7(a), 8(a) and $9(\mathrm{a})$, respectively. Each simulation conveys information of Long-Latcoordinate, time and sunspot-number. SNR(dB) in VOACAP simulation are based on $1 \mathrm{~Hz}$ bandwidth, however SNR by the WSPR observations are based on $3000 \mathrm{~Hz}$ receiver bandwidth, so we normalized the SNR-VOACAP by subtracting with a factor $35 \mathrm{~dB}$.
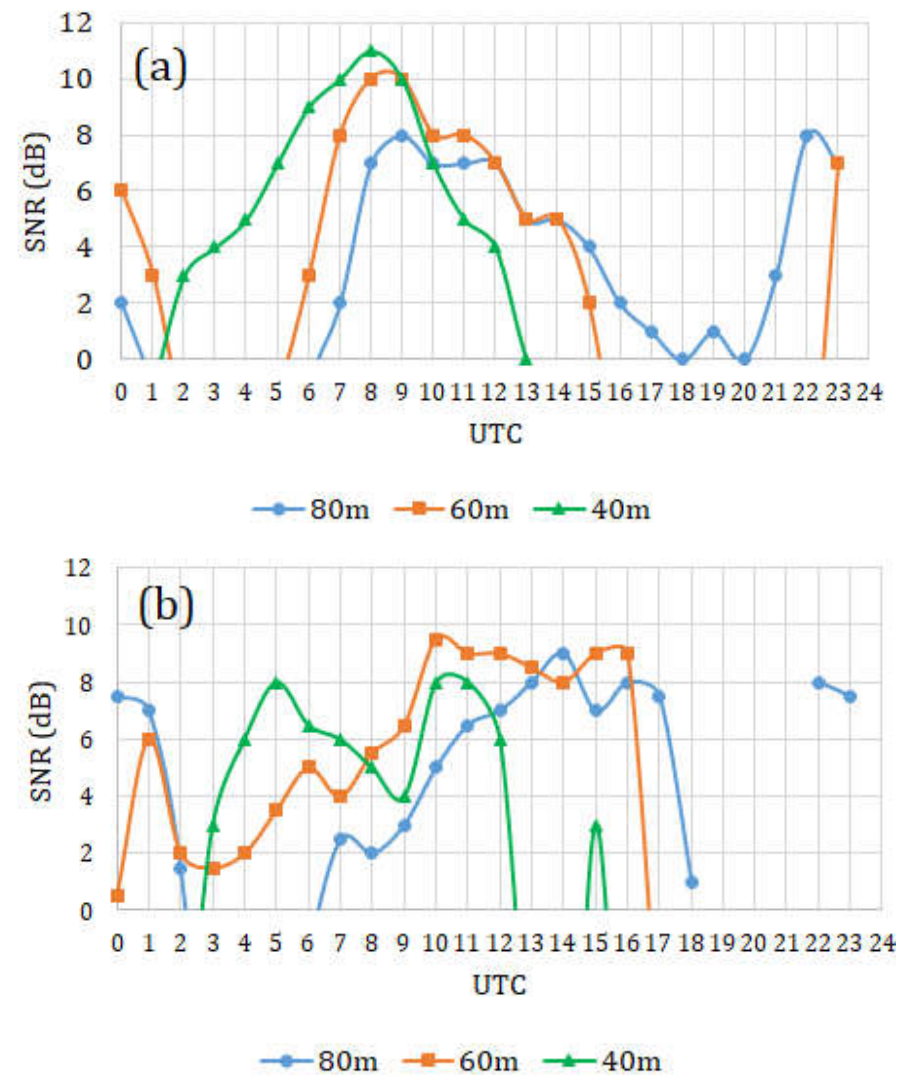

Figure 9. Results of measurement and simulation in January 6th 2018; (a) VOACAP simulation with SSN=8; (b) Measurement results.

Figure 7(b), 8(b) and 9(b) depict SNR above $0 \mathrm{~dB}$ of measurement results. Transmitter power during measurement was set to 10 watts, even though, all common $\mathrm{HF}$ communication radio have transmitted power 
specification until 100 watts. While, it is shown in those three Fig. 7, 8 and 9 that $60 \mathrm{~m}$ band has the longest NVIS channel availability between local sunrise and local midnight. Reliable voice HF communication require SNR more than $6 \mathrm{~dB}$ for operator grade, and more than $15 \mathrm{~dB}$ for public-used grade [18]. If the transmitter is set to 100 watts, then SNR increase with 10 $\mathrm{dB}$ up. Means, SNR of $60 \mathrm{~m}$ band reach over the requirements for establishing a reliable NVIS communication between local sunrise and local midnight.

\section{CONCLUSION}

How important the usage of sub-10 MHz HF band for humanitarian purposes have been analyzed by the regulatory consideration. Based on official views of attended Administration/State members during WRC-2015, could be concluded that they have the same spirit to use this sub-10 MHz band for PPDR and other humanitarian purposes, as the prominent usage.

The data of radio frequency usage licenses holder in the $3-10 \mathrm{MHz}$ band are listed on the Database Frequency Register managed by the Indonesian frequency authority (SDPPI). Data analysis and categorizing has been done. The results show that the dominant users are fixed service users. The number of those users are declining continuously. Means, the channels availability of frequency below $10 \mathrm{MHz}$ is getting better.

A simple propagation measurement by using WSPR sounding system have been done during the minimum solar cycle by using three working frequency in the band of $80 \mathrm{~m}, 60 \mathrm{~m}$ and $40 \mathrm{~m}$ respectively. The measurements have been done for three difference days with the approximately 3-months period. The comparison results between VOACAP simulation against WSPR measuring system shows that the measurement by using WSPR provide relatively a proper data that figure the instantaneous condition of NVIS channel.

Discrepancies between simulation and measurement are occurred mainly because of sporadic data in the band of $60 \mathrm{~m}$ and $80 \mathrm{~m}$. However, all of the measurement results and simulations almost have the same agreement regarding the quiet period between local midnight and local sunrise.

The results of measurements show that $60 \mathrm{~m}$ band is the most reliable NVIS channel between local sunrise and local midnight. Furthermore, 100 watts is a proper transmitter power to reach the required SNR for reliable voice communication.

The result of this study will become a platform of the recommendation to the Government, in order to decide a moratorium for a new fixed service frequency assignment for sub-10 $\mathrm{MHz}$ band, except only for the humanitarian and PPDR purposes. 


\section{Acknowledgements}

The authors would like to thank to the numerous people who are, and have been involved in this study. I am particularly grateful to my students Bustamir Arif and Rifqi Andi Setiawan for their support during observation precess; Mr. Kamal M Saleh (SDPPI) for his courtesy to share the official data of HF users; Ministry of Research and Higher Education Republic of Indonesia for their supporting via PTUPT research grant.

\section{REFERENCES}

[1] Witvliet A Ben, Alsina-Pages Ma Rosa, Radio Communication via Near Vertical Incidence Skywave Propagation: an overview, Telecommunication Syst, Springer, Online, Feb, 2017.

[2] Maslin Nicholas, HF COMMUNICATIONS: A SYSTEMS APPROACH, Pitman Publishing, pp. 63, 1987.

[3] ITU-R, Final Acts WRC-12, WORLD RADIOCOMMUNICATION CONFERENCE, 2012.

[4] ITU-R, Radio Regulation (RR), Article 5, Frequency Allocations, 2012.

[5] Law of The Republic of Indonesia On Telecommunication No.36 year 1999, Article 33, 1999.

[6] ITU-R, Radio Regulation (RR), Article 5, Frequency Allocations, 2015.

[7] Walden C Marcus, Comparison of propagation prediction and measurements for midlatitude $\mathrm{HF}$ near-vertical incidence sky wave link at 5 MHz, RADIO SCIENCE, VOL. 47, RSOL09, 2012.

[8] Zolesi Bruno, Cander $\mathrm{R}$ Ljiljana, Ionospheric Prediction and Forecasting, Springer Geophysics, p.44, p.185, 2014.

[9] Lane, G., Signal-to Noise Prediction Using VOACAP - User's Guide, Rockwell-Collins, Cedar Rapids, Iowa, 2001.

[10] ITU-R, Recommendations P. 533-13, Method for the prediction of the performance of HF circuits, 2015.

[11] Stocker A J., Fast and accurate calculation of multipath spread from VOACAP prediction, RADIO SCIENCE, Vol.47, RS 4005, 2012

[12] Witvliet A Ben, Maanen van Eric, Peterson J George, Measuring the Isolation of Circularly Polarized Characteristic Waves in NVIS Propagation, IEEE ANTENNAS \& PROPAGATION MAGAZINE, June, 2015.

[13] Gokya S Nicols, Understanding Propagation using WSPR to Gauge Propagation, RSGB Propagation Studies Committee, RADCOM, 2009.

[14] Dutono Titon, Ikeda Naomitsu, Watanabe Akira, Effect of Compound parameters on speaker-independent word recognition, J Acoustic Soc Jpn (E) 19, 1, pp 1-11, 1998.

[15] Dutono Titon Et al., A Spot Independent for NVIS Channel Observation, Proceeding of IES-ETA, Bali, pp. 247-251, 2018.

[16] ARRL, Antenna Handbook 2007, ARRL Publisher, Chapter 7, 2007.

[17] Ghasemi Abdolla, Abedi Ali, Farshid Ghasemi, Propagation Engineering in Wireless Communication, Springer, p.153-168, 2012 
[18] ITU-R, Recommendation ITU-R F.339-8, Bandwidth, signal-to-noise ratios and fading allowances in HF fixed and land mobile radiocommunication systems, 2013. 